strument is not therefore considered reliable for such purposes. In the use of this instrument, the chief disturbing influence is the effect of variation of latitude upon the earth's attraction, varying as the square of the sine of the latitude, the difference between the equatorial and polar attraction as established by pendulum observation being $\frac{1}{180}$ th of the former. The amount of this correction would be calculated as depth in fathoms and tabulated for use with the instrument.

The instrument would be chiefly useful in enabling the uariner to determine his position, when in foggy or cloudy weather he was unable to take observations. If the figure of the ocean bed was laid down more perfectly than at present upon charts, and such were in the hands of the mariner, he would be able to tell in observing his bathometer what was the approximate depth of water below him, and the direction in which, and the rate at which the depth either increased or diminished, while by consulting his chart he would then be enabled to determine his actual position with considerable accuracy.

\title{
DESCRIPTION OF AN IMPROVED FORM OF GOVERNOR FOR STEAM ENGINES OR OTHER PRIME MOVERS.
}

By William D. Marks, Ph. B., C. E., Member St. Louis Engineers' Club. Instructor in Mechanical Engineering, Lehigh University, Bethlehem, Pa.

In the writer's experience as a practical mechanic, his attention has often been called to the irregularities of the best forms of governors, whose action is dependent upon the centrifugal of the revolving pendulum; even after all that years of experience and most careful study have done for their greater accuracy of performance.

By this means he was led to give some thought to the requisites of a governor, which would be nearly, if not quite, isochronal in its action.

A careful study of the ordinary form of ball governor, convinced him that a governor such as was needed, must satisfy the following conditions :-

1. The motion of the valve must precede any change of speed in the governor balls; otherwise, as in the case of all centrifugal governors, the engine must go fast in order to go slow, or the reverse. Should the valve motion follow or be coincident with a change of 
speed in the governor, the engine's governor necessarily must vary for some interval of time, and the governor cannot be isochronous, because of a defect of principle. When, however, the valve motion can be made to precede a change of speed in the balls, the engine is so to speak, barred from any variation of speed upon making the attempt, and rendered practically isochronous.

2. The opening of the steam valve must be independent of the angle, which the arms attached to the balls, form with the central spindle, around which they revolve.

Thus an engine having its full amount of work, and governed by an ordinary ball governor, will be kept at a uniform speed by the governor so long as the average resistance to be overcome by the engine remains constant, but whenever any of the work is taken off, the speed of the engine will be increased to a higher rate, corresponding to the diminished work, and at this faster speed the engine will then run uniformly under the mastery of the governor, so long as the work continues without further alteration. This arises from the fact that the degree of opening of the steam valve is directly controlled by the angle to which the governor balls are raised by their velocity of revolution; the steam valve being moved only by a change of speed, and consequently by a change of the angle of suspension of the governor balls; whence it follows that a larger supply of steam for overcoming any increase of work can be obtained only, in conjunction with a smaller angle of the suspension rods of the governor balls, and consequently with a slower speed; and that a larger angle of the ball rods, and consequently a higher speed, must be attained in order to reduce the supply of steam for meeting any reduction of work to be done by the engine.

3. The governor must be sensitive, $i$. e., quick to act. This result is usually attained in the centrifugal governor, by giving to the balls a speed much greater than that of the engine, so that a slight variation of speed in the engine is multiplied in the governor many times.

A high speed, however, is attended with the disadvantage of rapid wear, and in the case of an ordinary governor, wear, such as to admit of any lost motion, is attended with much trouble to the engineer, and sudden variations of speed in the engine.

4. The governor must have power, which means an even and sure motion of the valve, notwithstanding the almost unavoidable defects of workmanship, such as the sticking of the valve, or the binding of the valve stem through careless packing of the stuffing box. In the 
ordinary governor this power is sought to be obtained either by a high speed, the defects of which have already been pointed out, or by means of very heavy balls, which results in a very cumbersome and large machine, besides adding largely to the expense.

5. Simplicity and economy must be attained in the construction and manufacture of the governor, otherwise the greater number of mechanics will prefer to use the ordinary form of governor, on account of its lesser cost-first cost often being an item much more regarded by mechanics than a true and intelligent spirit of economy would dictate.

A careful study of these before mentioned facts, shows that absolutely isochronous motion of the engine is unattainable, as the ability of the governor to regulate the speed of the engine is derived entirely from variations of the speed of the engine from any fixed rate, but it is quite possible to arrange so that the governor itself may be practically isochronous, and thus create a standard of speed to which the engine must conform, more or less nearly, according to the sensitiveness and power of the governor used.

This result I have endeavored to obtain by the use of the inertia of the balls of the free conical pendulum, in connection with a spiral spring and screw.

In this improved governor which I call isochronous, the pendulum spindle is in two sections, of which the lower one, to which, by means of a feather and key way, the driving power is geared, is capable of sliding lengthwise a little, at the same time that it turns the upper one, and screw up and down in the hub of its driving wheel; and is connected to the wheel by a spring which allows the wheel to over-run the spindle a little, when the motion increases to close the valve, in advance of any change of speed in the pendulum; and when the speed of the engine slacks, the tension of the spring will make the spindle over-run the wheel, which will screw the spindle along the other way, and thus open the valve in advance, of any change of speed in the pendulum; thus making a much more sensitive governor than others now in use.

The sensitiveness of this governor is determined by the flexibility of the spring used; the more flexible the spring, the more sensitive the governor to any variations of speed in the engine. The power to move the valve with evenness, is derived from the screw, the pitch of which determines its power. 
The spiral spring may have more than one coil, as shown in the drawing, which, while giving more sensitiveness to the governor, also gives greater power, because of permitting greater angular separation of the ends of the spring, and also admits of a less pitch to the screw, and consequently greater power to move the valve, when the distance, through which it is to be moved, is the same.

The figure shown is a partial sectional elevation; $A$ is the upper section of the spindle carrying the free conical pendulum $B ; C$

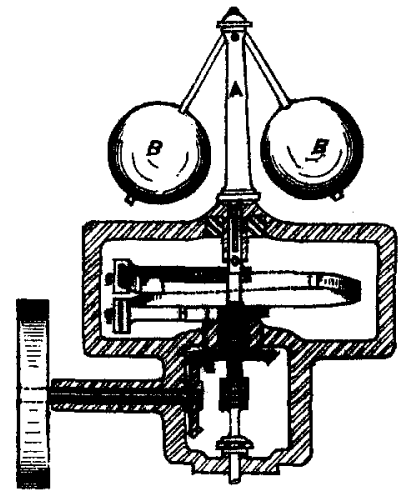
is the lower section to which the power through the spring $F$ is connected. It enters a socket $D$ in the upper section, and is capable of sliding lengthwise therein a little. It also carries an arm $E$, to the outer end of which is attached the upper end of the coiled spring $E$; whose other end is connected to the hub of the driving wheel $G$, by the $\operatorname{arm} H$; to allow the driving wheel to advance faster than the spindle for a short time, when the speed suddenly increases; and fall behind it when the speed slackens, in order to screw the spindle up or down by its threaded portion 1 , in the screw threaded hub of the driving wheel, and thus shift the valve up or down.

The power of this governor is only limited by the strength of the working parts, ball rods, spring, etc. Should the spring break under any unexpected strain, the immediate closing of the steam valve will result, as the screw threaded spindle will be at once screwed down to its fullest extent.

That this governor should have so great power, is not surprising, when it is seen that the inertia of the balls is used as a "point d'apui," from which the valve is worked, and that instead of making use of centrifugal force only, as hitherto, the whole inertia of the free conical pendulum is used. For the sake of clearness, it must be especially noted that the balls $B B$ are connected only to the central spindle $A$ by a pin at the top, and that their centrifugal force is not made use of in this governor.

The calculations of the dimensions of the governors, which are quite voluminous, are not added to this paper, as the principle can be clearly understood without formulæ by inspection of the drawing. 